\title{
Correction to: Adopting Quaternion Wavelet Transform to Fuse Multi-Modal Medical Images
}

\author{
Peng Geng ${ }^{1,2} \cdot$ Xiuming Sun ${ }^{3} \cdot$ Jianhua Liu ${ }^{4}$
}

Published online: 20 April 2018

(c) Taiwanese Society of Biomedical Engineering 2018

\section{Correction to: J Med Biol Eng (2017) 37:230-239 https://doi.org/10.1007/s40846-016-0200-6}

The article "Adopting Quaternion Wavelet Transform to Fuse Multi-Modal Medical Images", written by Peng Geng, Xiuming Sun, Jianhua Liu was originally published Online First without open access. After publication in volume [37], issue [2], page [230-239] the author decided to opt for Open Choice and to make the article an open access publication. Therefore, the copyright of the article has been changed to $($ )
The Author(s) [2018] and the article is forthwith distributed under the terms of the Creative Commons Attribution 4.0 International License (http://creativecommons.org/licenses/ by/4.0/), which permits use, duplication, adaptation, distribution and reproduction in any medium or format, as long as you give appropriate credit to the original author(s) and the source, provide a link to the Creative Commons license, and indicate if changes were made.

The original article can be found online at https://doi.org/10.1007/ s40846-016-0200-6.

Peng Geng

Gengpeng@stdu.edu.cn

1 School of Information Science and Technology, Shijiazhuang Tiedao University, Shijiazhuang 050043, China

2 Structure Health Monitoring and Control Institute, Shijiazhuang Tiedao University, Shijiazhuang 050043, China

3 Science Department, Zhangiiakou University, Zhangjiakou 075000, China

4 School of Electronic and Electric Engineering, Shijiazhuang Tiedao University, Shijiazhuang 050043, China 